Mejorar la calidad de la educación en escuelas de escasos recursos.

El caso de la Escuela Nueva en Colombia

Vicky Colbert de Arboleda 


\section{Resumen}

Escuela Nueva es una innovación en educación básica desarrollada en Colombia, que integra de manera sistémica y costo-efectiva estrategias curriculares, comunitarias, de capacitación, seguimiento y administración. Fue creada con el fin de ofrecer la primaria completa y mejorar la calidad de la educación en escuelas rurales del país, especialmente las multigrado. Su tarea primordial ha sido transformar la enseñanza convencional en la educación básica, a través de propuestas pedagógicas que privilegian el aprendizaje activo, participativo y cooperativo, el fortalecimiento de las relaciones escuela-comunidad y una estrategia de promoción flexible adaptada a las características y condiciones de la niñez más vulnerable. Esta promoción permite que los alumnos avancen de una unidad a otra y de un grado o nivel a otro, a su propio ritmo. Escuela Nueva surgió como innovación local, a mediados de la década de 1970, y llegó a convertirse en política nacional a finales de la de 1980 cuando se implementó en más de 20.000 escuelas rurales. Mediante procesos continuos de evaluación y seguimiento al desarrollo del aprendizaje, en particular, y de la experiencia, en general, ha conseguido mejorar la calidad de la educación en Colombia y en múltiples países en donde se ha adaptado y contextualizado, lo cual se ha evidenciado a través de estudios de evaluación realizados por diversos organismos nacionales e internacionales.

\section{Palabras clave}

Escuela Nueva, aprendizaje significativo, aprendizaje cooperativo, currículo transversal, constructivismo, escuela multigrado, comportamiento democrático, calidad en la educación, microcentro, metodologías de aprendizaje.

\section{Abstract}

Escuela Nueva (New School) is a primary education innovation developed in Colombia to integrate curriculum, community, teaching, follow-up and management strategies systemically and cost-effectively. It was created in order to offer complete elementary education and improved quality in rural schools of the country, especially in multigrade situations. Its main task has been to transform traditional teaching methodologies for elementary schools, through diverse pedagogical proposals that privilege active, participative and cooperative learning, the strengthening of community-school relationships, and flexible promotion strategies that respect the conditions and characteristics of the most vulnerable children. Escuela Nueva appeared as a local innovation in the mid 70 s and became national policy by the late 80 s when it was implemented in more than 20000 rural schools. Through permanent evaluation and follow-up processes for the development of learning and experience in itself, the quality of education has been improved in Colombia as well as in those countries where this model has been adapted and contextualized. This has become evident through diverse studies and evaluations performed by national and international institutions

\section{Key words}

Escuela Nueva, educational innovation, learning guides, cooperative learning, democratic behavior, quality of education. 


\title{
Mejorar la calidad de la educación en escuelas de escasos recursos. El caso de la Escuela Nueva en Colombia*
}

\author{
Vicky Colbert de Arboleda'
}

\section{Introducción}

La deficiencia en la calidad de la educación es una de las amenazas más grandes para América Latina a pesar de que existen innovaciones que demuestran cómo mejorar calidad y equidad.

El sistema de la Escuela Nueva es un buen ejemplo de una innovación local y departamental de mediados de los años setenta del siglo XX, la cual se convirtió en una política nacional colombiana y se desarrolló en la mayoría de las escuelas rurales a finales de la década de 1980. Muchos de sus principios, elementos y estrategias se han introducido en escuelas urbanas y en la básica secundaria en escuelas rurales. Además, inspiró aspectos de la Ley de Educación y varias reformas educacionales en el orden mundial.

Gracias a la combinación de varios cursos con uno o dos maestros - cuando la situación multigrado lo requiere--, al aprendizaje cooperativo, al apoyo niño a niño, la adopción de nuevos métodos de enseñanza activos centrados en la participación de los niños, un nuevo rol del maestro como orientador y facilitador del aprendizaje y un nuevo concepto de guías de aprendizaje o textos interactivos, Colombia ha sido capaz de proveer una educación primaria completa y mejorar su calidad en regiones de bajo nivel socioeconómico.

\footnotetext{
* Texto recibido el 9 de noviembre de 2006, evaluado y arbitrado el 11 de diciembre de 2006.

${ }^{1}$ Coautora del modelo pedagógico Escuela Nueva (conjuntamente con Óscar Mogollón) y fundadora y actual directora ejecutiva de la Fundación Escuela Nueva Volvamos a la Gente®, ong sin ánimo de lucro creada en 1987 por el grupo fundador y autor del modelo pedagógico Escuela Nueva y por expertos de amplia trayectoria en el reconocimiento de los problemas sociales y educativos de Colombia, con el fin de cualificar y fortalecer procesos educativos en comunidades rurales y urbanas de bajos recursos económicos.vcolbert@volvamos.org
} 
Escuela Nueva transforma la escuela convencional; es una innovación de educación básica que integra de manera sistémica y costo-efectiva estrategias curriculares, comunitarias, de capacitación y formación docente, de gestión y administración, e involucra niños, docentes, comunidades y agentes administrativos.

El punto de partida de su propuesta conceptual y metodológica es que se requiere un nuevo paradigma de aprendizaje, de una "nueva escuela" y un enfoque de una "escuela abierta" para mejorar la efectividad y calidad de las escuelas rurales en zonas de escasos recursos económicos. Como resultado, Escuela Nueva puso en práctica principios válidos de teorías modernas de aprendizaje a través de aprendizajes colaborativos y participativos, y demostró que las prácticas pedagógicas tradicionales, transmisivas y pasivas podían cambiarse masivamente hacia un nuevo paradigma pedagógico basado en el aprendizaje cooperativo, personalizado, comprensivo y constructivista. Logró modificar el modelo educativo estándar, centrado en el docente, hacia un modelo participativo y cooperativo basado, de manera prioritaria, en el estudiante.

El Primer Estudio Internacional Comparativo de la Unesco, Laboratorio Latinoamericano de Evaluación de la Calidad de la Educación 1999, comparó once países y encontró que el único donde el estrato rural está mejor que el urbano oficial, excepto megaciudades, es Colombia, gracias a la Escuela Nueva. Varios organismos internacionales y la literatura mundial sobre educación hacen referencia a esta experiencia como una de las innovaciones educativas más importantes de los últimos años y modelo para otros países en desarrollo.

Seis aspectos importantes deben resaltarse sobre esta experiencia colombiana:

- Demostró que se puede lograr un mejoramiento cualitativo y cuantitativo en escuelas de bajos recursos económicos.

- Logró modificar masivamente el modelo frontal de enseñanza, centrado en el profesor, a un modelo centrado en el niño, con un aprendizaje activo, participativo y cooperativo, y un cambio del rol del docente.

- La situación multigrado forzó a todo el sistema a innovar en las prácticas pedagógicas, los procedimientos de evaluación del aprendizaje, las políticas de textos y en la formación docente. Inspiró la nueva ley de educación.

- Evidenció que el aprendizaje cooperativo puede iniciar cambios en comportamientos democráticos, en la convivencia pacífica y en el aprendizaje participativo desde una temprana edad. Así mismo, desarrolló valores, actitudes y habilidades para una ciudadanía activa.

- Es una de las innovaciones que ha logrado mayor sostenibilidad; las innovaciones son muy vulnerables a cambios administrativos y políticos.

- Tiene un modelo marco suficientemente flexible que puede ser tomado en consideración en culturas y sociedades diferentes, permitiendo su adaptación a varios países como Brasil: Escola Activa; Guatemala: Escuela Nueva 
Unitaria; Panamá: Escuela Activa; Chile: Mece Rural; El Salvador: Aulas Alternativas; México: Escuela Interactiva Comunitaria; Nicaragua: Escuela Modelo; República Dominicana: Escuela Multigrado Innovada; Perú: Aprendes; Guyana: Escuela Nueva; Uganda: New School; Filipinas: Active School, Honduras: Escuela Activa Participativa.

\section{Situación de la educación básica primaria²}

\section{En Colombia}

El diagnóstico educativo (DNP, 1975, p. 6) sintetizaba que en las zonas rurales se generaba un sistema educativo deficiente, reflejado en varios aspectos: precarios accesos al sistema, baja valoración de la educación como mejoramiento, baja calidad de la instrucción impartida y entrada temprana de los niños al mundo del trabajo.

La inequitativa distribución de los recursos del Estado entre el sector rural y urbano había llevado a que en 1974 el 48,4\% de la población entre 7 y 14 años no estaba escolarizada en el campo, mientras que en la ciudad era de 22,5\%; la deserción en el nivel de primaria para el sector rural era del $72 \%$, mientras que en el sector urbano estaba entre el 38 y $33 \%$. (...) Adicionalmente, mientras que la población urbana en edad escolar era cubierta en un $90 \%$ en las zonas rurales, se atendía menos del $40 \%$ y sólo el $20 \%$ de los que se matriculaban en primer grado llegaban a quinto grado, debido a repitencia excesiva y la alta deserción; se evidenciaba una gran ineficiencia en el sistema educativo en el nivel de primaria, en la medida en que el grado de escolaridad de la zona urbana era de 3,7 años y la situación de escolaridad en la zona rural apenas alcanzaba 1,7 años (Ministerio de Educación Nacional, 1998: 24 y 39).

Entre los factores responsables de este panorama crítico de la educación primaria en el sector rural aparecen las mismas causas generalizadas de la educación colombiana en escuelas de bajos recursos económicos.

Según Colbert (2006), las principales causas de las altas tasas de fracaso escolar en escuelas de bajos recursos son:

- Metodologías tradicionales, pasivas, de clase magistral, que hacen énfasis en la memorización antes que en la comprensión y no dan relevancia al desarrollo de habilidades de pensamiento superior, difíciles de aplicar en escuelas de escasos recursos donde hay una alta heterogeneidad de edades y extraedad, y niños con diferentes ritmos de aprendizaje debido al ingreso

\footnotetext{
2 Para mayor información se puede ver el texto de Wolff, Lawrence; Schiefelbein, Ernesto y Valenzuela, Jorge (1993), Resultado de la educación primaria para América Latina y El Caribe.
} 
tardío, la repitencia y la extraedad. El método frontal responde más a las condiciones y características del alumno promedio, pero los menos capacitados tienen que repetir el grado la mayoría de las veces porque no pueden seguir el ritmo del estudiante medio. Son pocas las clases que motivan a los estudiantes para aprender.

- Los planes y programas de estudios son sobrecargados y tienen poca relación con la vida diaria del alumno.

- Poco tiempo asignado en el primer grado a actividades de aprestamiento para el logro de destrezas básicas en lectoescritura y cálculo elemental.

- Dificultades en la transición del niño de la familia a la escuela formal.

- Carencia de textos y materiales educativos apropiados y coherentes con metodologías activas y participativas, y con las características y necesidades de las escuelas (por ejemplo, escuelas multigrado).

- Calendarios y sistemas de promoción y evaluación rígidos.

- Escaso tiempo dedicado al aprendizaje efectivo.

- Capacitación de maestros poco efectiva, es decir, que no mejoran sus prácticas pedagógicas en el aula. Sólo algunos maestros han participado en un proceso de capacitación activo y vivencial y en temas prioritarios según las características y necesidades de las escuelas.

- En la zona rural hay un gran porcentaje de escuelas monodocentes y bidocentes con maestros que no han sido capacitados en estrategias para atender la escuela multigrado.

- Las escuelas situadas en zonas geográficamente aisladas no atraen maestros calificados.

- Hay un traslado permanente de maestros a los centros urbanos.

Todos los diagnósticos realizados por el DNP coincidian en la necesidad de ampliación de cobertura con especial énfasis en las áreas rurales y mejoramiento de la calidad de la enseñanza primaria. Es así como el Plan de Universalización ampliaría la aplicación masiva de los resultados de programas como Escuela Nueva. Este Plan pretendía universalizar el servicio educativo a todo el país.

Colombia, así como otros países de Latinoamérica, experimentó una expansión acelerada en la educación primaria a principios de 1950. Como resultado, la cobertura total llegó casi al 80\% en 1994, pero la distribución de esta cobertura revela grandes y marcadas diferencias entre algunas regiones del país y entre las zonas urbana y rural. Mientras que la cobertura en el área urbana alcanza un 89\%, en el área rural es de sólo un 66\%. La calidad y la eficiencia de la educación básica pública, tanto en los niveles de primaria y secundaria, son extremadamente pobres. Los estudiantes de menores ingresos son los más afectados. 


\begin{abstract}
Algunos selectos programas nacionales, en particular la Escuela Nueva, han hecho significativas contribuciones al extender el acceso a la educación a los más pobres. Durante el período del proyecto de universalización de la educación primaria (1988-1996) ha habido un incremento del 45,6\% en las escuelas rurales, mientras que en las urbanas el aumento fue de 7,6\% (Chacón, Gómez, Losada, Rodríguez y Rojas, 1992).
\end{abstract}

\title{
En América Latina
}

La región ha progresado en cuanto a ampliar el acceso, pero aún prevalece una educación primaria ineficiente y de baja calidad que conduce a altos niveles de fracaso y educación incompleta.

En cuanto al rendimiento en el aprendizaje, los resultados en la región son sustancialmente inferiores a los obtenidos por los países desarrollados, e inferiores a muchos de los países asiáticos en desarrollo. Además, las pruebas de rendimiento de aprendizaje demuestran que los alumnos a duras penas logran el $50 \%$ de lo esperado en el currículo oficial vigente, y que sólo la mitad de los alumnos de cuarto grado comprende lo que lee. De hecho, incluso el promedio de los alumnos de la elite de las escuelas privadas no alcanza el promedio nacional de los países desarrollados.

El ingreso tardío en el sistema, la repitencia y la deserción, temporal o permanente, disminuyen la tasa neta de matrícula y, por tanto, el número de niños que logra completar la primaria.

Una consecuencia del ingreso tardío en el sistema y de la alta repitencia es una mayor heterogeneidad en edades, lo cual es bastante notorio en las zonas rurales y urbano-marginales, precisamente donde las escuelas tienen las tasas más altas de fracaso escolar. La heterogeneidad afecta negativamente el aprendizaje en clase, en especial cuando los maestros utilizan métodos tradicionales de instrucción, dado que para producir ritmos de aprendizaje diferentes se requieren estrategias de aprendizaje personalizado y grupal.

\section{La necesidad de una asegurar una educación básica de calidad y una renovación pedagógica como elemento central para garantizar equidad, en las oportunidades de acceso y éxito en el sistema educativo}

Como lo plantea la Unesco, el acceso a la primaria es ya casi universal en la región, pero ha sido más difícil mejorar la calidad de los procesos de aprendizaje porque ello demanda un cambio cultural (Unesco/Orealc, 1996). "Se requiere un cambio sustancial en los conceptos de educación y conocimiento que incluya la capacidad de comprender lo que se ha aprendido, desarrollar capacidades, conocimientos e información en la vida diaria, aprendiendo a convivir en una sociedad más compleja". 
Esta visión de la educación representa un gran desafío en una región en la que la instrucción se ha basado en la enseñanza frontal, llamada bancaria por Paulo Freire.

Existe un consenso creciente sobre la necesidad de ofrecer una buena educación - entendida como una síntesis del conocimiento, la comprensión, el saber hacer-, y la capacidad de convivir con otros, como una condición necesaria para que Colombia se pueda beneficiar de las oportunidades que se abren con el actual milenio.

Un desarrollo sostenible y equitativo sumado a la consolidación de las democracias requieren cambios en la calidad y en la equidad de la educación. Entre estos cambios sobresale uno esencial que es avanzar desde el énfasis en transmisión de contenidos hacia la construcción de aprendizajes grupales y colaborativos.

Estos planteamientos indican que los esfuerzos realizados en los últimos años han generado cambios institucionales y un gran énfasis en reformas de carácter administrativo.

Si bien no existen respuestas a preguntas como: qué va primero, la renovación institucional y de gestión o la pedagógica, la hipótesis que ha prevalecido en la última década ha sido que la mejor estrategia de cambio consiste en actuar sobre los modelos de gestión y administración del sistema. A pesar de los esfuerzos realizados en esta línea, este énfasis no ha provocado mejores logros de aprendizaje, especialmente en los alumnos de bajos recursos económicos. Ahora es el momento para una renovación pedagógica, métodos, contenidos y formación y capacitación docente.

Las declaraciones mundiales de Jomtien y Dakar, las metas del milenio y el movimiento de Educación para todos (EFA) le han dado prioridad a la educación básica. En estas conferencias mundiales, todos los gobiernos del mundo reiteraron su compromiso renovado de asegurar la educación básica como la base para un aprendizaje y un desarrollo humano permanentes sobre el cual los países pueden construir sistemáticamente nuevos niveles y nuevos tipos de educación y capacitación (Conferencia Mundial de Educación para Todos, 1990).

La Declaración de la Conferencia de Jomtien definió las necesidades básicas de aprendizaje como

las herramientas esenciales para el aprendizaje y los contenidos básicos del aprendizaje necesarios para que los seres humanos puedan sobrevivir, desarrollar plenamente sus capacidades, vivir y trabajar con dignidad, participar plenamente en el desarrollo, mejorar la calidad de su vida, tomar decisiones fundamentales y continuar aprendiendo (Conferencia Mundial de Educación para Todos, 1990).

El desafío consiste en definir aquello que es posible enseñar realmente en la escuela y que los alumnos pueden aprender y aplicar más tarde en su vida. 
La alta repitencia, la baja retención y los bajos puntajes en los estudios de medición de logros de aprendizaje en América Latina sugieren que, aun cuando muchos desearían que en la escuela se enseñaran múltiples conocimientos y habilidades, necesitamos definir unos objetivos básicos y realistas para la educación primaria, y especialmente para las escuelas de escasos recursos que son las que presentan el más alto fracaso escolar. Ernesto Schiefelbein, ex ministro de Educación de Chile y ex director de la Oficina Regional de Educación para América Latina de la Unesco plantea que esto significa que por lo menos los niños aprendan a: i) leer comprensivamente, ii) comunicarse en forma escrita y oral, iii) resolver y aplicar operaciones de aritmética elemental, y iv) practicar comportamientos democráticos, v) observar y aprender de su propia realidad, vi) resolver problemas de la vida diaria (Schiefelbein, 1993).

De igual manera, Víctor Ordóñez, ex director general de Educación Básica de la Unesco afirma que para mejorar la calidad de la educación en América Latina no es suficiente hacer énfasis sólo en la expansión de los sistemas educativos actuales: no se puede seguir ofreciendo más de lo mismo (Ordóñez, 1995).

Así mismo, el profesor Joseph Farell, profesor emérito y ex director del Instituto de Educación de la Universidad de Toronto, sostiene que los estudios comparativos demuestran que las reformas educativas presentadas desde los niveles centrales no están dirigidas a solucionar los más graves problemas de las escuelas de escasos recursos. Hay un reconocimiento muy difundido de que se debe tomar a la escuela como unidad fundamental de cambio y que es esencial realizar cambios en las prácticas de enseñanza y del aprendizaje en el aula. El mejoramiento de las prácticas pedagógicas en el aula requiere una revisión profunda de la educación básica a la luz de un nuevo paradigma educativo, el cual centra al niño como sujeto activo que participa en su propio proceso de aprendizaje. Conceptos tales como creatividad, aprendizaje cooperativo, aprendizaje activo y participativo, solución de problemas, participación con la comunidad, aparecen constantemente. Sin un cambio en el aula, la expansión cuantitativa del sistema educativo producirá más de lo mismo. Más y más niños recibirán una educación de mala calidad que los preparará deficientemente para responder a las exigencias del desarrollo tecnológico, social, económico, cultural y político (Unesco, 1991).

Los principios del nuevo paradigma están ampliamente fundamentados en las corrientes pedagógicas de principios de siglo y en las teorías modernas de aprendizaje. Lo novedoso es aplicarlos por medio de estrategias concretas en escuelas de escasos recursos. El sistema de Escuela Nueva en Colombia ha operacionalizado estos principios en estrategias operativas y costo-efectivas para poder transformar las escuelas de más bajos recursos económicos en ambientes de aprendizaje activos, participativos y colaborativos.

La escuela convencional donde un profesor enseña a un alumno promedio (método frontal de enseñanza) limita la posibilidad de ofrecer una educación de buena calidad. 
La expansión de la oferta, hasta dar acceso a la escuela a toda la población, ha evidenciado el carácter heterogéneo de los grupos que ingresan en los sistemas educativos y la falta de flexibilidad de la educación frontal-tradicional para dar una respuesta educativa adecuada a la gran heterogeneidad de edades, culturas y a la pluralidad lingüística. Por esta razón, los elementos innovadores tienen que basarse en métodos de enseñanza renovados, aprendizaje personalizado y trabajo grupal.

Retomando lo anterior, el profesor Ernesto Schiefelbein afirma: "Sólo una revisión profunda de la educación básica, teniendo en cuenta un nuevo modelo de escuela y métodos de enseñanza renovados, permitirán lograr la calidad requerida para el siglo XXI, y así responder a los requerimientos de los procesos democráticos y al desarrollo social y económico de nuestros países" (Schiefelbein, 1993).

\section{La Escuela Nueva de Colombia}

(Colbert, Chiappe y Arboleda, 1993)

\section{Esfuerzos previos: escuelas multigrado y la metodología de la Escuela Unitaria}

En Colombia, como en muchos países desarrollados y en vías de desarrollo, existen las escuelas multigrado, en especial en zonas de baja densidad de población donde uno o dos maestros trabajan simultáneamente con todos los cursos de la básica primaria.

De acuerdo con las investigaciones educativas, la organización de una escuela multigrado requiere un mayor grado de innovación. Escuelas de este tipo precisan modificar las prácticas tradicionales de enseñanza y desarrollar un proceso de aprendizaje más centrado en el niño. Un maestro que maneje varios cursos a la vez, debido a diferentes ritmos de aprendizaje y a la heterogeneidad presente en los salones, encuentra necesario organizar a los estudiantes en pequeños grupos, introducir un aprendizaje cooperativo y desarrollar estrategias personalizadas y flexibles. Estas estrategias de enseñanza demandan materiales especialmente diseñados para el aprendizaje independiente y el trabajo cooperativo.

La Escuela Nueva fue creada como respuesta a los persistentes problemas de la educación rural colombiana, y se organizó a partir de las bases de la Escuela Unitaria promovida por la Unesco, la cual trató de dirigirse hacia los problemas educativos del sector rural de países en vías de desarrollo. Buscó los mismos objetivos pero introdujo nuevas estrategias operativas que permitieran una mayor viabilidad técnica, política y financiera.

La escolaridad incompleta, las altas tasas de deserción escolar y de repetición caracterizaban en ese entonces a las escuelas del sector rural como aquellas donde se daba la mayor inequidad e ineficiencia del sistema educativo. El altísimo fracaso escolar estaba causado en parte por las metodologías tan pasivas y me- 
morísticas, pocas clases motivaban a los estudiantes, un currículo sobrecargado y poco pertinente a su vida diaria, carencia de materiales educativos apropiados, sistemas rígidos de evaluación y promoción que expulsaban y no retenían a los estudiantes, escuelas aisladas geográficamente y maestros desmotivados buscando trasladarse a las ciudades.

Adicionalmente, muchos niños y niñas participaban en las cosechas, sobre todo en la zona cafetera, y migraban de unas zonas a otras. Cuando regresaban a la escuela tenían que repetir el año. La escuela no se estaba adaptando a las condiciones de vida de estos niños; se requerían estrategias más flexibles, adaptadas a las condiciones de vida del niño campesino.

Lo anterior demostraba que se necesitaba un cambio profundo en la escuela primaria y que no se podía seguir ofreciendo más de lo mismo. Más y más niños y niñas recibirían una educación de mala calidad que los preparaba deficientemente para responder a las exigencias del desarrollo tecnológico, social, económico, cultural y político.

Adicionalmente, la mayoría de las escuelas en el sector rural eran y son multigrado, y estas escuelas demandan una intervención específica. La escuela multigrado es un fenómeno mundial existente tanto en países desarrollados (Suecia, Noruega, Canadá, Australia, Francia, entre otros) como en vías de desarrollo, que existen prioritariamente en zonas de baja densidad de población.

Estas escuelas eran y son invisibles a los planificadores de la educación y a las instituciones formadoras de docentes, y requieren mayor innovación y estrategias específicas para manejar diferentes ritmos de aprendizaje en el aula, para manejar la heterogeneidad. Precisan romper con el modelo convencional de enseñaza centrado en un docente e introducir estrategias personalizadas y de trabajo colaborativo entre los niños, apoyados por materiales especialmente diseñados para el aprendizaje independiente y el trabajo en grupo. En esas condiciones las metodologías tradicionales y convencionales, de clase magistral y frontales, no son una respuesta.

El Primer Proyecto Principal en América Latina, auspiciado por la Unesco entre 1960 y 1970, promovió la Escuela Unitaria, y recomendaba a los ministros la organización de estas escuelas a fin de ofrecer la primaria completa en los países.

A partir de una declaración en una reunión de ministros de Educación llevada a cabo en Ginebra, Suiza, en 1961, oficialmente se apoyó a las escuelas unitarias de todo el mundo. Éstas permitían que uno o dos maestros dictaran la primaria completa, especialmente en zonas de baja densidad poblacional, de modo que el o los maestros podían trabajar simultáneamente con varios grados y grupos de alumnos de diferentes edades y distintos niveles de desarrollo. Las principales características de la Escuela Unitaria eran: enseñanza individualizada, fichas de aprendizaje elaboradas por los maestros, promoción automática y múltiples tableros. Era el comienzo de la introducción de estrategias personalizadas. 
Infortunadamente, la Escuela Unitaria, en su proceso de expansión, generó resistencia de los docentes porque creían que era una forma de eliminar docentes (desconocían la realidad mundial de la escuela multigrado, donde para poco número de alumnos no se requieren tantos docentes) y por el exceso de trabajo al elaborar ellos mismos las fichas individualizadas. La respuesta a estos problemas y la introducción de estrategias más operativas y viables dio el nacimiento a la Escuela Nueva, que pretendía ir más allá de la connotación de monodocencia e introducir, a partir de la escuela multigrado, una renovación de las prácticas pedagógicas en todas las escuelas rurales.

\section{La Escuela Nueva}

Es un sistema de educación primaria iniciado en Colombia, el cual integra estrategias curriculares, administrativas, comunitarias y de capacitación para los docentes. El programa fue diseñado en 1976 con el fin de proveer una educación primaria completa y mejorar la efectividad de las escuelas rurales de la nación, en especial las multigrado.

Este sistema proporciona un aprendizaje activo, una relación más estrecha entre la escuela y la comunidad, y un mecanismo flexible de promoción adaptado al estilo de vida del niño campesino. La promoción flexible permite a los estudiantes avanzar en un curso y terminar unidades académicas, según su propio ritmo de aprendizaje.

Adicionalmente, promueve un proceso de aprendizaje cooperativo y personalizado centrado en el alumno, la formación de valores y comportamientos democráticos, un nuevo rol del docente como orientador y facilitador y un nuevo concepto de textos interactivos o guías de aprendizaje para lograr así el mejoramiento de la calidad y efectividad del aprendizaje.

La Escuela Nueva se inició bajo dos supuestos fundamentales. El primero era que para introducir cambios en el niño, se necesita también innovar en las estrategias de capacitación de los docentes, en el trabajo con la comunidad, e innovar en la estructura administrativa y de gestión. De igual forma, el sistema ofrece una respuesta integral mediante el desarrollo de cuatro importantes componentes básicos y presenta estrategias concretas para los niños, maestros, la comunidad y los agentes administrativos. En segundo lugar, asume que es esencial desarrollar mecanismos que sean replicables, descentralizados y financiera, técnica y políticamente viables. En otras palabras, el diseño del sistema debe incluir estrategias que faciliten replicar procesos "e ir" a escala nacional.

La Escuela Nueva estimula:

- Un aprendizaje centrado en el niño.

- Un currículo relevante basado en la vida cotidiana del niño.

- Un calendario flexible así como sistemas de evaluación y nivelación.

- Una mejor relación entre la escuela y la comunidad. 
- Énfasis en la formación de valores participativos y democráticos.

- Prácticas y estrategias efectivas de capacitación para los maestros.

- Una nueva generación de guías o textos interactivos de aprendizaje, coherentes con el aprendizaje cooperativo y personalizado.

Sus principales objetivos son los de inculcar ciertas actitudes y habilidades en los estudiantes, maestros, administradores y demás miembros de la comunidad. Algunos de ellos son los siguientes:

Para los estudiantes: promover: un activo, reflexivo y participativo proceso de aprendizaje; la habilidad de aplicar conocimientos a nuevas situaciones; un mejorado autoconcepto; actitudes cooperativas y democráticas, y una serie de habilidades básicas en matemáticas, lenguaje y ciencias sociales y naturales.

Para los maestros: facilitar el deseo de servir como guía y facilitador en vez de ser sólo un transmisor de información; fomentar: la habilidad de ser un líder de la comunidad; una actitud positiva hacia el trabajo en el entorno rural y en la Escuela Nueva; una actitud positiva hacia los administradores y directivos; técnicas de instrucción de la Escuela Nueva y habilidades en la instrucción multigrado.

Para los agentes administrativos: estas escuelas procuran promover una relación colaborativa y orientadora entre docentes y los agentes administrativos, en vez de una rígida y controladora, y la habilidad de manejar efectivamente la capacitación para los maestros.

Para la comunidad: impulsar una solidaria relación con los profesores, niños y la comunidad local. La Escuela Nueva ofrece a los padres de familia, parientes y al resto de la comunidad la oportunidad de participar en actividades escolares y revitalizar su cultura local a través de las actividades y de los instrumentos de la escuela.

La Escuela Nueva toma la escuela como la unidad de cambio para mejorar la calidad de la educación. Permite la provisión de una completa educación primaria, especialmente en donde existe una escolaridad incompleta o deficiente, y facilita una aproximación a una perspectiva multigrado en donde lo requiera la situación - uno o dos maestros manejando todos los cursos de primaria simultáneamente-, así como un mejoramiento cualitativo en escuelas pobres, tanto urbanas como rurales.

La Escuela Nueva como sistema, programa y metodología, que nace en Colombia en 1975, se inspira en el movimiento pedagógico Escuela Nueva-que surgió en las últimas décadas del siglo XIX y comienzos del siglo XX-, y que rompió con la educación tradicional pasiva y cambió el concepto de maestro que dicta clases, con un enfoque memorístico y autoritario, a un educador nuevo que asume como centro de acción pedagógica al alumno y que utiliza nuevos métodos para el aprendizaje activo centrado en el niño o la niña.

Concretamente, pedagogos y pensadores como Decroly, Dewey, María Montessori, Piaget y Vygotsky sirvieron de inspiración. La filosofía no es nueva; lo 
nuevo fue ponerlo en práctica masivamente en escuelas de bajos recursos económicos y no sólo en colegios de elite. Se integró todo aquello que se sabe mejora la calidad de una manera sistémica y costo-efectiva.

En el sistema de Escuela Nueva hay varios aspectos básicos del aprendizaje que están en sintonía con el constructivismo que propicia la construcción social del conocimiento a través del diálogo y la interacción. Los profesores pasan, por ejemplo, de transmisores de conocimientos a ser facilitadores, interactúan con la comunidad, basan el conocimiento en el contexto local y amplían el ámbito del aprendizaje para incluir logros en el comportamiento social. También existen muchos elementos de inteligencias múltiples.

El aprendizaje activo participativo, colaborativo y personalizado caracteriza el sistema de Escuela Nueva, y la interacción social es un elemento esencial del proceso de aprendizaje. Se le otorga un papel central al pensamiento del niño, de manera que propicie la construcción de conocimientos en trabajo en pequeños grupos; establece una relación directa entre el aprendizaje y el medio ambiente físico y comunitario; propicia y respeta el ritmo de avance durante los procesos de construcción de conocimientos; cuenta con estrategias pedagógicas y didácticas que promueven y facilitan en los niños la construcción de conocimientos y promueve el desarrollo personal y social en contextos democráticos y autónomos a través de los gobiernos estudiantiles.

En la orientación del currículo de la Escuela Nueva se tiene en cuenta su pertinencia desde los puntos de vista social y cultural, así como las experiencias de aprendizaje activo y participativo para los niños.

Los elementos del componente curricular utilizados para orientar el aprendizaje en el sistema Escuela Nueva son: las guías de autoaprendizaje o textos interactivos para los niños, una biblioteca escolar, rincones o centros de aprendizaje y la organización de un gobierno estudiantil de los escolares.

Un elemento importante del componente curricular es la guía de aprendizaje de autoinstrucción o textos interactivos del estudiante. Éstos promueven un aprendizaje cooperativo y activo centrado en el estudiante, vinculan experiencias de aprendizaje con la familia y la comunidad, y estimulan el desarrollo de habilidades de pensamiento superior y mecanismos de promoción flexible donde se avanza a diferentes ritmos de aprendizaje.

Las guías de aprendizaje promueven la construcción colectiva de conocimientos por parte de los niños al igual que facilitan el trabajo individual o en equipos con estrategias de ayuda de niño a niño; también sirven como herramientas de planeación y adaptación curricular para el profesor. Son organizadas por secuencias y desarrollan los temas fundamentales de los temas y programas de estudio.

Otra característica importante de las guías es que combinan un currículo nacional con posibilidades regionales de cambio o adaptación hecha por los maestros. 
También son elementos del currículo los rincones de aprendizaje, que facilitan la manipulación de material concreto para promover el aprendizaje comprensivo y no memorístico, además de que sirven de vehículo para articular la escuela con la cultura local y la comunidad; la biblioteca-aula para complementar y apoyar las actividades de aprendizaje y para estimular el aprender a investigar, y el gobierno estudiantil de los niños, que sirve para iniciarlos en actitudes y comportamientos democráticos y cívicos. Los niños son organizados en comités, y éstos pueden ser vinculados a grupos comunitarios. Igualmente existen instrumentos específicos que catalizan procesos de participación y de desarrollo socioafectivo; se articulan con la formación de valores.

La participación de la comunidad en actividades escolares es otro elemento clave. Pretende la integración entre estudiantes y profesores en las actividades escolares, y promueve acciones que beneficien tanto a la escuela como a la comunidad. Ejemplos de algunos instrumentos útiles para apoyar el trabajo incluyen un croquis de la vereda, fichas familiares y un calendario agrícola. Estos instrumentos son adicionalmente útiles para la elaboración de los Proyectos Educativos Institucionales, PEI.

En el componente de capacitación y seguimiento los docentes son formados para utilizar y aplicar los elementos del currículo de la Escuela Nueva en la organización del aula y de la comunidad, y en la utilización y adaptación de las guías de aprendizaje al alcance de los estudiantes, de la comunidad, y el entorno local cuando sea necesario. Su principal objetivo es cualificar y recuperar el verdadero papel del profesor para guiar, orientar y evaluar el proceso de aprendizaje, evitando gastar tiempo dando instrucciones rutinarias. Son capacitados por medio de talleres locales secuenciales y replicables, y utilizan módulos o guías de aprendizaje para los docentes que siguen metodologías similares a aquellas que posteriormente utilizarán con sus alumnos. Los talleres están más orientados hacia la práctica que hacia lo teórico-académico, y son complementados con círculos de docentes o microcentros donde los docentes reflexionan sobre sus prácticas pedagógicas y se colaboran mutuamente. Son espacios que promueven la construcción colectiva de conocimiento.

Las escuelas demostrativas permiten que los maestros observen las escuelas en plena actividad para promover nuevos comportamientos y actitudes hacia el mejoramiento de sus prácticas pedagógicas. Por medio de los talleres locales de seguimiento, los microcentros, los docentes interactúan y aprenden de otros más experimentados, comparten sus prácticas de enseñanza y resuelven problemas concretos.

\section{La participación social en la Escuela Nueva}

La participación es la dimensión fundamental y transversal de todos los componentes del sistema. Cada elemento de los componentes incorpora o incluye un proceso participativo. Por ejemplo, las guías o textos interactivos requieren una 
continua interacción entre los estudiantes, padres de familia, docentes y otros miembros de la comunidad educativa. Estas múltiples formas de interacción conducen a una construcción social del conocimiento.

El gobierno escolar de los alumnos es, por definición, la herramienta que permite a los estudiantes desarrollar valores relacionados con la democracia, la solidaridad, la disciplina y, en general, el cumplimiento de los derechos y deberes de la vida comunitaria. La elección del gobierno estudiantil, presidente, vicepresidente, secretario y tesorero, y de varios integrantes de los distintos comités, el buzón de sugerencias, el autocontrol de asistencia, el libro de participación, el cuaderno viajero, entre otros, son todos instrumentos que infunden y motivan a la participación directa y activa por parte de los estudiantes.

Las herramientas que comprometen a los padres de familia y a los demás miembros de la comunidad también exigen un alto nivel de participación. Por ejemplo, la elaboración conjunta entre estudiantes, alumnos y comunidad, del croquis o mapa de la vereda, y la organización de los rincones de trabajo con elementos y recursos traídos por los mismos niños de sus casas.

La estrategia y el proceso de capacitación de los maestros también son muy participativos. Se inicia con observación directa e interacciones con otros docentes en las escuelas demostrativas. Cada uno de los talleres requiere la participación activa de cada docente para aprender mediante prácticas y aplicar lo que aprendieron. Los microcentros son autodirigidos por los docentes y se realizan periódicamente para compartir sus aprendizajes, con lo que se fortalece el principio de colaboración.

\section{Dificultades en el proceso de expansión masiva}

Este proceso de expansión masiva a finales de la década de 1980, coincidió con la nueva política de descentralización del país. El Ministerio de Educación utilizó toda su energía en su propia reorganización en el momento en que la expansión de la Escuela Nueva a todas las escuelas requería mayor capacidad organizacional y gerencial para la entrega de los servicios. Surgieron muchos problemas de gestión y administración, como la distorsión de la estrategia de capacitación, la improvisación en el entrenamiento y en los capacitadores, el regreso a la formación tradicional, la no articulación de los eventos de ésta con la entrega de materiales y la poca preparación en el manejo de los mismos. Adicionalmente, se perdió el seguimiento y el apoyo a los docentes y se incorporaron nuevos maestros y administrativos sin la capacitación requerida. Muchas escuelas sólo se quedaron con el nombre y no implementaron todas las intervenciones de la Escuela Nueva. Surgieron críticas por los problemas administrativos y de gestión, y hubo confusión entre estos aspectos con el modelo marco. Finalmente, un considerable número de maestros capacitados fueron trasladados a los sectores urbanos por parte de los alcaldes, quienes ahora tenían el poder administrativo sobre los docentes. El efecto final es que el Ministerio 
de Educación abandonó su prioridad en las escuelas rurales por muchos años, y los municipios no desarrollaron estrategias de gestión para apoyar la Escuela Nueva.

En la nueva institucionalidad, una necesidad urgente es establecer nuevas alianzas con la sociedad civil y buscar mecanismos de gestión local para que los municipios, los nuevos actores, las comunidades y otros sectores como los de salud, medio ambiente, comunicaciones, agricultura y cultura apoyen, cualifiquen y se apropien de la innovación.

A pesar de todos los problemas de gestión mencionados, los resultados de la Escuela Nueva hablan por sí solos: continúan teniendo mejores logros académicos y socioafectivos en los niños, no sólo en Colombia sino comparativamente con otros países de Latinoamérica. Ésta es una de las razones por las cuales 35 países se han inspirado en esta experiencia, tanto para conocer el modelo como para comprender las estrategias de cambio por las cuales una innovación puede sobrevivir, pese a las deficiencias del sector educativo.

\section{Lecciones aprendidas}

Algunas de las lecciones aprendidas sobre sus estrategias de cambio y de implementación son las siguientes:

- El proceso usó un enfoque de abajo hacia arriba que tuvo en cuenta a la escuela como unidad de cambio.

- El proceso debe ser gradual y bien monitoreado en lugar de masivo y vertical.

- Hubo una fuerte alianza con la sociedad civil. En este caso la Federación Nacional de Cafeteros ha sido un gran aliado. Muchos de los mejores casos son implementados en la zona cafetera.

- El éxito de la Escuela Nueva es mayor en las regiones donde las comunidades se involucraron, los círculos de aprendizaje de los docentes continuaron, los maestros hicieron adaptaciones locales y se apropiaron del proceso.

- La existencia de guías de aprendizaje, conocidas y manejadas por los niños, ha permitido que el proceso pedagógico se mantenga y sea implementado de manera adecuada. Irónicamente, muchos niños han sido capaces de orientar a los nuevos maestros que han llegado a las escuelas sin ningún entrenamiento previo.

- El hecho de que hubo un esfuerzo de evaluación constante ha mantenido el interés en la escuela como un modelo de innovación.

- La presencia constante de organismos internacionales ha contribuido a mantener la escuela en movimiento.

- El enfoque sistémico ha permitido que por lo menos algunos de los componentes y elementos funcionen cuando otros no lo hacen, y generen cambios positivos, aun si son pequeños, en la escuela. 
- También ha sido importante el sostenimiento y la promoción continuos por parte de los miembros del equipo original, quienes a pesar de muchos cambios y circunstancias adversas han logrado mantener viva la innovación ${ }^{3}$.

- La demanda, la aplicación y la adaptación exitosas en otros países, muestra que el modelo marco de la Escuela Nueva puede inspirar y ser adaptado a otros contextos.

En conclusión, se puede plantear la hipótesis de que cuando la dimensión transversal de participación, o la noción de alianzas se tomó en consideración en la implementación del sistema, la innovación sobrevivió y continuó desarrollándose. Éste fue claramente el caso en el departamento de Caldas, donde la Secretaría de Educación estableció una alianza con el Comité de Cafeteros. Bajo el liderazgo del Comité, la Escuela Nueva se mantuvo en el departamento, mejoró y enriqueció el componente comunitario al establecer el gobierno estudiantil de los niños y jóvenes a las necesidades de la comunidad, y extendió la metodología a los niveles de posprimaria. Esto ha facilitado que el departamento haya organizado un modelo de educación básica completa en las áreas rurales utilizando el enfoque y las estrategias de la Escuela Nueva.

Otro ejemplo que apoya esta hipótesis es la prevalencia de los microcentros o círculos de estudio locales de los docentes en los departamentos de Quindío y Antioquia. El principio de colaboración, al permitir que los docentes reflexionen sobre sus propias prácticas pedagógicas, ha estimulado a los docentes de estas regiones, donde es común encontrar microcentros que han estado funcionando por años de manera voluntaria.

\section{Evaluaciones más importantes de la Escuela Nueva}

Distintas evaluaciones realizadas por organismos nacionales e internacionales desde 1980 demuestran que la Escuela Nueva mejora, significativamente, los estándares educativos de la primaria rural y urbana, y la autoestima de niños y niñas. Entre estas evaluaciones se destacan las promovidas por las siguientes entidades:

- Colciencias, 1982

- Instituto SER de Investigación, 1987

- Evaluación cualitativa de educación realizada por el grupo noruego Imtec, 1990

- Psacharopoulos, Vélez y Rojas - Banco Mundial, 1992

\footnotetext{
${ }^{3}$ La Fundación Escuela Nueva Volvamos a la Gente, organización no gubernamental constituida en 1988 por varios ex ministros de Educación y el equipo técnico fundador de la Escuela Nueva en Colombia innovaron en estrategias para el mejoramiento de la calidad de la educación y fortalecieron la alianza entre el Estado y la sociedad civil.
} 
- Análisis de datos de Pruebas Saber, 1993

- Misión Social del Departamento Nacional de Planeación, 1994

- Universidad de Stanford - Patrick McEwan, 1995

- Laboratorio Latinoamericano de la Calidad de la Educación, Unesco, 1998

- Escuela Nueva's impact on the peaceful social interaction of children in Colombia, de Clemente Forero en Education for All and Multigrade Teaching. Challenges and Opportunities. Angela Little (ed.). Institute of Education, University of London. Springer, 2006.

La Misión Social del Departamento Nacional de Planeación de Colombia, en su revista Planeación y Desarrollo No. 28 de 1997 concluye que: "El resultado más relevante en educación es que los logros de Escuela Nueva en el nivel socioeconómico 1 son mayores que los de la escuela tradicional, incluso en escuelas de nivel socioeconómico 2; Escuela Nueva compensa las limitaciones iniciales de los niños, ocasionadas por bajo nivel socioeconómico" (Departamento Nacional de Planeación, 1997).

El Primer Estudio Internacional Comparativo de la Unesco, liderado por el Laboratorio Latinoamericano de Evaluación de la Calidad de la Educación en 1998, después de la aplicación de pruebas en lenguaje y matemáticas en once países, concluyó que "Colombia es el único país de la región en donde la escuela rural obtiene mejores resultados que la escuela urbana, exceptuando mega ciudades". Adicionalmente, demostró que "en educación rural, en matemáticas sólo Cuba está por encima de Colombia”.

En la comparación de once países, el único en que el estrato rural está mejor que el urbano es Colombia, prioritariamente por Escuela Nueva (Unesco, 1998).

\section{GRÁFICO 1. LA CALIDAD DE LA EDUCACIÓN EN COLOMBIA ESTÁ CERCA} DEL PROMEDIO DE LA EDUCACIÓN EN AMÉRICA LATINA

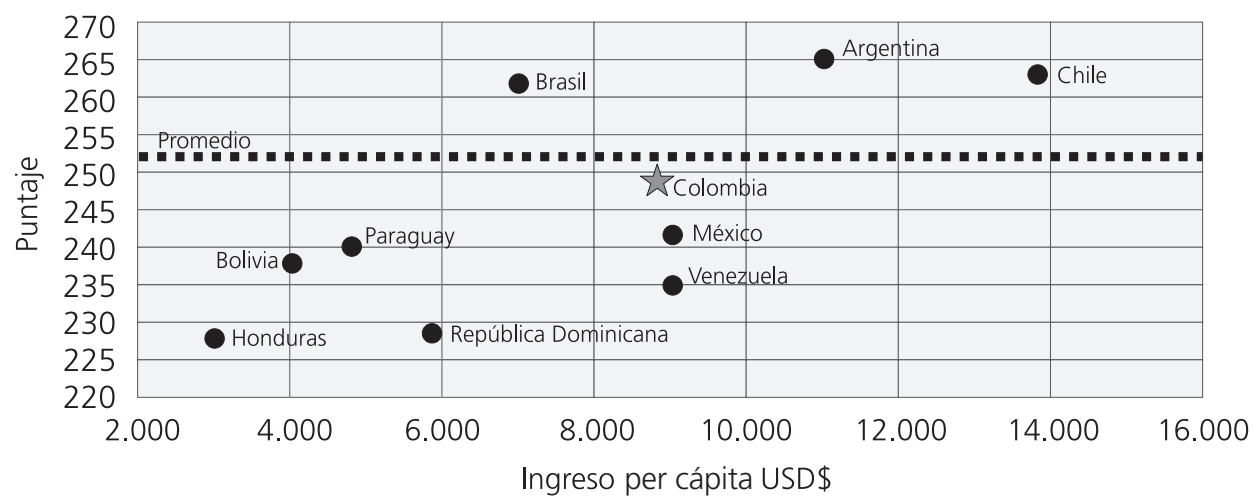

Fundación Escuela Nueva Volvamos a la Gente 
GRÁFICO 2. LA EDUCACIÓN RURAL EN COLOMBIA TIENE MEJOR CALIDAD QUE LA EDUCACIÓN URBANA (Excepto grandes ciudades en América Latina)

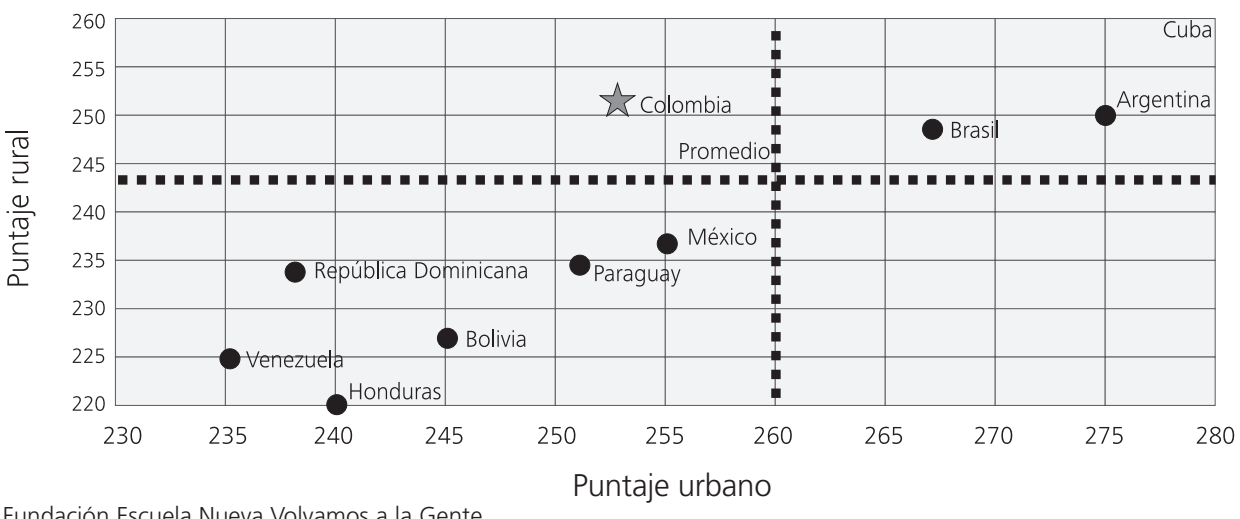

Fundación Escuela Nueva Volvamos a la Gente

GRÁFICO 3. EN MATEMÁTICAS SÓLO CUBA ESTÁ POR ENCIMA DE COLOMBIA (Educación rural)



Fundación Escuela Nueva Volvamos a la Gente

En las Pruebas Saber de Colombia, aplicadas por el MEN en los años 2002 y 2003 para evaluar los distintos modelos educativos, tanto en matemáticas como en lenguaje los resultados de Escuela Nueva superaron de manera significativa los promedios nacionales y los de los otros modelos de básica primaria.

\section{Escuela Nueva, convivencia pacífica y comportamiento democrático}

El aprendizaje cooperativo puede iniciar cambios en el comportamiento democrático y en la convivencia pacífica desde una edad temprana y, de acuerdo con Johnson y Johnson (1987), está demostrado que reduce prejuicios y conductas agresivas, promueve el comprender diferentes perspectivas, fortalece la autoestima y estimula el desarrollo de la solidaridad, la tolerancia y la aceptación de las diferencias. 
El aprendizaje colaborativo en la Escuela Nueva ha reconfirmado estos planteamientos sobre esta estrategia de aprendizaje, y tal como lo ha planteado José Bernardo Toro, "las rutinas pedagógicas orientadas al trabajo de grupo, participación, auto-aprendizaje, tienen mejor oportunidad de formar un ethos democrático que aquellas que son meramente directivas".

En 2002, un estudio sobre comportamiento democrático y convivencia realizado por la Universidad del Rosario de Colombia y la Fundación Escuela Nueva Volvamos a la Gente, y publicado recientemente por la Universidad de Londres, determinó "la superioridad de las Escuelas Nuevas sobre las Escuelas Convencionales en la formación de comportamientos de convivencia y democracia en la niñez". Escuela Nueva demuestra significativos resultados en la formación del comportamiento democrático y la interacción social pacífica de los niños, en comparación con escuelas convencionales.

Las conclusiones principales del estudio han confirmado lo siguiente:

- La escuela sí tiene influencia en el desarrollo del comportamiento democrático y en destrezas de interacción social pacífica en los niños y las niñas.

- El impacto de las escuelas es significativo, aun en ambientes de violencia.

- Hay un importante impacto directo del sistema de la escuela en las prácticas de las familias de los estudiantes y es aquí donde Escuela Nueva difiere más de las escuelas convencionales.

- La probabilidad de que los padres perciban un impacto de la escuela en las prácticas de la casa crece con el nivel de implementación de Escuela Nueva.

El estudio de comportamiento democrático (Academia para el Desarrollo de la Educación, 1994) realizado en Guatemala por la Academy for Educational Development, AED, evidencia resultados similares; los niños y niñas de las Escuelas Nuevas Unitarias dan mayor retroalimentación positiva y menor retroalimentación negativa entre ellos, que aquellos de las Escuelas Unitarias Tradicionales. Adicionalmente, tienen frecuencias más altas tomando turnos y liderando procesos dentro de la escuela.

\section{Fundación Escuela Nueva Volvamos a la Gente, ejemplo de alianza público-privada para promover la calidad y sostenibilidad del modelo}

Previendo que las innovaciones son muy vulnerables a los cambios políticos y administrativos, en 1987 se creó la Fundación Escuela Nueva Volvamos a la Gente, organización no gubernamental sin ánimo de lucro creada por el grupo fundador y autor del modelo pedagógico Escuela Nueva y por expertos de amplia trayectoria en el reconocimiento de los problemas sociales y educativos de Colombia, con 
el fin de promover la calidad y sostenibilidad de Escuela Nueva y de cualificar y fortalecer procesos educativos con este sistema en comunidades rurales y urbanas de escasos recursos económicos.

Igualmente, en un esfuerzo por fortalecer Escuela Nueva en Colombia y el exterior, ha liderado la organización y realización de dos Congresos Internacionales de Escuelas Nuevas. El primero se llevó a cabo en el año 2003 en Armenia, Colombia, y más recientemente, del 2 al 4 de marzo de 2006, se desarrolló la segunda versión de este importante evento en Medellín, Colombia, con 1.400 participantes de más de 15 países.

Los objetivos del evento fueron:

- Compartir los avances de Escuela Nueva en Colombia y otros países.

- Socializar los logros de otras experiencias educativas inspiradas y desarrolladas a partir de Escuela Nueva.

- Compartir los desarrollos de Escuela Nueva en nuevos contextos y poblaciones escolares de alta vulnerabilidad.

- Hacer explícito cómo Escuela Nueva promueve competencias básicas y ciudadanas.

De esta manera, mediante innovaciones e iniciativas de esta índole, la Fundación Escuela Nueva Volvamos a la Gente contribuye al mejoramiento, a la cobertura, la calidad, la eficiencia y la sostenibilidad de la educación básica rural y urbana, a través de Escuela Nueva, prioritariamente en países en desarrollo, por medio de alianzas público-privadas.

\section{Adaptación de Escuela Nueva al contexto urbano. Escuela Nueva Activa}

En 1988, la Fundación Escuela Nueva Volvamos a la Gente, con el apoyo de la Fundación Interamericana, inició la adaptación de Escuela Nueva a sectores urbanos, denominándola Escuela Nueva Activa. En la década de 1990, Escuela Nueva se expandió gradualmente a diversas ciudades de Colombia.

Evaluaciones sobre competencias básicas en lenguaje y matemáticas, realizadas por la Universidad Nacional de Colombia entre los años 2000 y 2002 a 20 instituciones educativas urbanas de Bogotá demostraron incrementos significativos de 40,36\% en lenguaje y 69\% en matemáticas después de la implementación de las estrategias de Escuela Nueva realizadas por esta fundación.

\section{Adaptación de Escuela Nueva a nuevos contextos y poblaciones vulnerables y migrantes}

Ante la problemática del desplazamiento interno de Colombia causado por el conflicto armado, existen 3.100.000 desplazados desde 1985. De éstos, el 54\% 
corresponde a menores de 18 años y el 30\% de ellos es menor de cinco años. El $91 \%$ ha sido desplazado de municipios pequeños y áreas rurales, mientras que el 9\% ha sido desplazado de ciudades capitales. El 55\% del total migra a las ciudades capitales, siendo éstas las de mayor concentración de población desplazada (Codhes, 2005).

Esta población en edad escolar migrante que proviene de zonas de violencia requiere un sistema educativo adaptable y flexible a su situación de movilidad: flexible en procesos de aprendizaje y en requerimientos de matrícula. Precisa apoyo socioafectivo adicional y personalizado para fortalecer su autoestima, estabilidad familiar, sensibilidad en el manejo de la heterogeneidad cultural y de edades, y mayor énfasis en destrezas básicas de aprendizaje, destrezas sociales y un aprendizaje más amigable, dado el fracaso escolar acumulado.

Las limitaciones de la escuela convencional ante las necesidades de esta población migrante son las siguientes: un modelo de enseñanza frontal centrado en el docente magistral, en el que el conocimiento es transmitido, se asume que todos los estudiantes aprenden y avanzan al mismo ritmo, se tiende a homogeneizar, se tiene un espacio limitado para la atención personalizada y el diálogo y la interacción entre los estudiantes y sus familias; se da un débil desarrollo de destrezas sociales y un mayor énfasis en resultados académicos. Por todas estas razones podemos plantear la hipótesis de que las escuelas convencionales no se adaptan a las necesidades y condiciones de la niñez desplazada y migrante; tienden a expulsarlos, en vez de atraerlos y retenerlos.

La decisión de formular y ejecutar esta iniciativa se sustenta en las siguientes razones:

- Por los resultados probados de Escuela Nueva, tanto en lo rural como en lo urbano.

- La mayoría de los estudiantes que han migrado del campo a las ciudades han estado familiarizados con alguno de los componentes de Escuela Nueva.

- Las características, los componentes, las estrategias y los elementos de Escuela Nueva responden más a una educación transformativa basada en un enfoque de derechos de la niñez y que promueve:

Aprendizaje participativo y colaborativo fomentado por Escuela Nueva; el aprendizaje se da a través del diálogo y la interacción.

Un currículo pertinente.

Un sistema de promoción flexible.

Fortalecimiento de relación escuela-comunidad.

Énfasis en desarrollo de comportamientos sociales y democráticos.

Estrategias de capacitación y seguimiento efectivas.

Currículo modularizado con guías de autoaprendizaje interactivas.

Costo-efectividad y replicabilidad. 
- Su ambiente de aprendizaje es amigable y flexible y se adapta a su situación de movilidad y a la heterogeneidad etaria y cultural.

En 2002, la Fundación Escuela Nueva Volvamos a la Gente diseñó e inició el pilotaje en el año 2003 de adaptar el modelo pedagógico de Escuela Nueva a estas poblaciones (inicialmente se denominó Escuela Nueva Itinerante), financiado por la Usaid y apoyado por la Oficina Internacional de Migraciones y el Ministerio de Educación Nacional de Colombia.

A una segunda fase se unen el Consejo Noruego para Refugiados. El objetivo principal de este convenio era incorporar al sistema educativo a la niñez excluida, respetando su derecho a una educación, y los objetivos más específicos han sido: la creación de ambientes de aprendizaje amigables que operan en la misma comunidad y son parte del sistema formal; la dotación de guías de autoaprendizaje pertinentes e integradas con énfasis en destrezas básicas para la vida; la recuperación de la confianza y la autoestima de la niñez y el deseo de aprender y la incorporación de niños en extraedad.

En 2004 y 2005, con pruebas de Unesco/Orealc se evaluó la adaptación de Escuela Nueva para niños y niñas desplazados a través de los Círculos de Aprendizaje-Escuela Nueva Activa. Evidenciaron una mejoría en lenguaje y matemáticas del 36, 1\% y 30,4\% , respectivamente. "La relación del grado $5^{\circ}$ de los círculos con el promedio nacional de lenguaje $(69,7 \%)$ es de 13,9 puntos por encima $(83,6 \%)$ y de matemáticas $(52 \%)$ de $17,3 \%$ puntos por encima $(69.3 \%)$ ".

La autoestima de los niños y niñas se incrementó en un 18,5\%. En mayo, el $76 \%$ de los menores tenía autoestima normal, $22 \%$ baja y el $2 \%$ muy baja (requerían atención terapéutica). En noviembre, seis meses después, el 94,5\% de los niños presenta autoestima normal, 5,52\% baja y ninguno requiere atención terapéutica.

\section{Hechos sobresalientes}

\section{y conclusiones de Escuela Nueva}

\section{Orden nacional}

- Logró que por primera vez en Colombia se ofreciera la primaria completa en escuelas rurales de uno o dos maestros, donde antes sólo se ofrecían los tres primeros niveles de primaria.

- A través de las investigaciones que realizaron Colciencias, el Instituto SER de Investigación, el Banco Mundial y la Unesco, la Universidad de Stanford, el Departamento de Planeación Nacional, la Universidad de Londres y en las Pruebas Saber, a pesar de que en la década de 1990 no tuvo todos los insumos requeridos para su adecuado funcionamiento, falta de materiales, capacitación de maestros, Escuela Nueva demostró logros superiores en los niños. 
- Está inspirada en una válida teoría educativa moderna y modelos de aprendizaje, y evidencia que se puede lograr masivamente un cambio del modelo pedagógico tradicional y "frontal" a uno nuevo basado en la participación y el aprendizaje activo y en un cambio del rol del docente.

- También promovió que por primera vez el Estado dotara gratuitamente de textos y bibliotecas a las escuelas rurales.

- Los elementos que constituyen el modelo, como por ejemplo el gobierno escolar, tienen un potencial para dinamizar una educación para la democracia, la participación, y así como la vida cívica, los instrumentos de trabajo con la comunidad promueven la participación de los padres y la comunidad alrededor de la escuela.

- Propició, por primera vez, un espacio de participación horizontal para la capacitación y el seguimiento de los maestros rurales mediante la creación de los microcentros de capacitación.

- Inspiró la creación de consejos estudiantiles y gobiernos escolares, y la aplicación de la escuela activa en todo el país para la parte urbana bajo la nueva ley de educación.

- Inspiró la posprimaria del Comité de Cafeteros de Caldas y la promovida por el Ministerio de Educación Nacional.

- La Misión de Sabios en 1994 de la Misión de Ciencias, Educación y Desarrollo recomendó su apoyo y difusión.

- El Informe de las Naciones Unidas sobre Desarrollo Humano entregado en enero de 2000 seleccionó a Escuela Nueva como uno de los tres logros más importantes del país.

\section{Orden internacional}

- Fue seleccionada por el Banco Mundial, 1988-1989, como una de las tres experiencias más sobresalientes en el mundo de los países en desarrollo que ha demostrado éxito a escala nacional, a partir de una iniciativa local.

- Varios organismos internacionales, Unesco, Unicef, OEA, AID, BID, Banco Mundial y la literatura mundial sobre educación, hacen referencia a esta experiencia como una de las innovaciones educativas más importantes de los últimos años y modelo para otros países en desarrollo.

- La mayoría de países de la región (República Dominicana, Brasil, Guatemala, Honduras, México, Costa Rica, Ecuador, Perú, Bolivia, Salvador, Paraguay, Panamá, Nicaragua y Chile) están desarrollando adaptaciones de Escuela Nueva, con la asesoría de profesionales colombianos. 
Las escuelas multigrado pueden llegar a ser invisibles para los planeadores educativos, tal como lo dice Angela Little: "Quienes diseñan, patrocinan y manejan los sistemas escolares nacionales, los sistemas de capacitación a los maestros, el desarrollo curricular o de materiales o sistemas de asesoría, ignoran esta realidad" (Little, 1996).

En el caso de Colombia, en donde más de 60\% de las escuelas rurales son multigrado, la administración central de la nación asume que las prevalecientes son escuelas monogrado. De lo aprendido respecto a por qué la Escuela Nueva ha sobrevivido y ha tenido un campo de acción tan fuerte, a pesar de las debilidades del sistema, se conoce que en su mayor parte se debe al proceso de cambio e implementación de esta reforma educativa, a la naturaleza de los currículos y de la innovación pedagógica y al verdadero cambio e impacto que ha causado en las actitudes de maestros y estudiantes.

El hecho de que las innovaciones educativas fueran iniciadas en un proceso de abajo para arriba, especialmente al transformar el contenido curricular y los métodos pedagógicos, explica en parte la razón por la cual la Escuela Nueva sigue viva y causando un gran impacto.

De acuerdo con el artículo "Escuela de Gobierno de la Universidad de Harvard" escrito por Michael Drabble (1999) sobre la Escuela Nueva, el creciente enfoque de pequeños y efectivos cambios en vez de unas "megarreformas", el hecho de que "pequeñas soluciones" fueran introducidas en el trabajo diario de los maestros y el empoderamiento del personal de la escuela fueron esenciales para el proceso de cambio de abajo hacia arriba y la continua sobrevivencia de la Escuela Nueva.

El hecho de que los actores reales de cambio sean niños, docentes y demás miembros activos de la comunidad implica que esta dimensión debe continuar y ser un eje central en el futuro; debe ser fortalecida para permitir su continua evolución y asegurar su sostenibilidad.

El modelo marco de la Escuela Nueva, con sus dos principales énfasis en el aprendizaje y la participación, continuamente evoluciona en lo local gracias a los esfuerzos de docentes y comunidades creativas. También evoluciona en el orden nacional en respuesta a los cambios sociales y económicos y a las nuevas necesidades; especialmente hay que establecer nuevas alianzas con otros sectores y con la sociedad civil y complementar la gestión del Estado e incorporar nuevos actores de las comunidades. En el orden internacional, aun cuando en el modelo marco se aplican muchas diferencias sociales y culturales, lo enriquecen y se integran a él. Así mismo, las nuevas tecnologías de información y comunicación permiten mayor interacción entre docentes y planificadores. En resumen, la naturaleza flexible y dinámica de la Escuela Nueva, promovida hace más de veinte años, puede todavía percibirse como pionera para el siglo XXI. 


\section{Bibliografía}

Academia para el Desarrollo de la EduCACión, AED. (1994). Indicadores de la Escuela Nueva Democrática en Escuelas Nuevas Unitarias, ENU. Guatemala, Proyecto de Investigación.

Chacón, C., Gómez, M., Losada, R., Rodríguez, P. y Rojas, L. (1992). Evaluación del Plan de Universalización de la Educación Básica Primaria, informe final. (pp. 89-91). Bogotá: Instituto SER de Investigaciones, documento IFT-252.

Colbert, V. (Marco de referencia) (2006). Hacia una Nueva Escuela para el Siglo XXI. Guías de formación docente en estrategias para el mejoramiento de la Educación Básica Primaria y para el aprendizaje personalizado y colaborativo. Fundación Volvamos a la Gente. Bogotá: Quebecor World.

Chiappe, G. y Arboleda, J. (1993). The New School Program. More and Better Primary Education for Children in Rural Areas in Colombia. En: Effective Schools in Developing Countries. London: The Falmer Press.

, Mogollón, Ó. y Levinger, B. (1974). Manual de capacitación docente. Primera publicación. Derechos reservados de autor. Bogotá: Ministerio de Educación Nacional.

et al. (2006). Hacia una Nueva

Escuela para el Siglo XXI. Guías de formación docente en estrategias para el mejoramiento de la Educación Básica Primaria y para el aprendizaje personalizado y colaborativo. Fundación Volvamos a la Gente. Bogotá: Quebecor World.
Conferencia Mundial de Educación Para Todos. (1990). Satisfacción de las necesidades básicas de aprendizaje. Nueva York (p. 158). Documento de referencia, WCEFA.

Departamento Nacional de Planeación. (1997). Planeación y desarrollo. Volumen XXVIII. Bogotá: Misión Social.

Drabble, M. (1999). Escuela Nueva (pp. 5-6). John F. Kennedy School of Government, Harvard University.

Forero, C. (2006). Escuela Nueva's impact on the peaceful social interaction of children in Colombia. En: Angela Little (ed.), Education for All and Multigrade Teaching: Challenges and Opportunities.

Johnson, D.; Johnson, F. (1987). Joining together: Group theory and group skills. 3rd ed. Englewood Cliffs, NJ: Prentice-Hall.

LitTle, A. (1996). Multigrade Teaching, a Review of Research and Practice. Dordrecht. London: Overseas Development Administration.

InSTITUTE OF EdUCATION. University of London. (2006). En: Angela Little (ed.), Education for All and Multigrade Teaching: Challenges and Opportunities.

Ministerio de Educación Nacional de Colombia. (1984). Plan de fomento para la educación en el área rural y los centros menores de población. Bogotá. . (1998). Educación para la población rural: balance prospectivo (p. 24). 
Schiefelbein, E. (1993). En busca de la escuela del siglo XXI. ¿Puede darnos la pista la Escuela Nueva de Colombia? Chile: Unesco. Unicef.

\section{Consultoría para los Derechos} Humanos y el Desplazamiento, CoDHES. (2005). Sistema de información sobre desplazamiento forzado y derechos humanos. Sidhes.

UNESCO. (1991). Informe final cuarta reunión del Comité Regional Intergubernamental del proyecto principal de la esfera de la educación en América Latina y el Caribe (p. 20). Quito.
/Oreala. (1996). Educación para el desarrollo y la paz: valorar la diversidad y aumentar las oportunidades de aprendizaje personalizado y grupal.

Wolff, L., Schiefelbein, E. y Valenzuela, J. (1993). Resultado de la educación primaria para América Latina y el Caribe: aprendizaje, grados aprobados y repetición. En: Mejoramiento de la calidad de la educación primaria en América Latina y el Caribe: hacia el siglo XXI. Banco Mundial, América Latina y el Caribe, Programa de Estudios Regionales, Informe 28. 